INPLASY

PROTOCOL

To cite: Song et al. Efficiency between Guan-fu base A versus propafenone in the treatment of arrhythmia: a meta-analysis. Inplasy protocol 202130077. doi:

10.37766/inplasy2021.3.0077

Received: 22 March 2021

Published: 22 March 2021

Corresponding author:

Jinming Song

928128995@qq.com

Author Affiliation:

Tianjin University of Traditional

Chinese Medicine

Support: None.

Review Stage at time of this submission: The review has not yet started.

Conflicts of interest:

None declared.

\section{Efficiency between Guan-fu base A versus propafenone in the treatment of arrhythmia: a meta-analysis}

Song, J1; Tang, Y2; Gao, C3; Xu, Z4

Review question / Objective: P: Patients with arrhythmia including patients with supraventricular or ventricular arrhythmia or patients with ventricular arrhythmia without severe structural heart disease or other diseases. I/C: Guanfu base $(4 \mathrm{mg} / \mathrm{kg}$, iv) A or propafenone (PRO, $1 \mathrm{mg} / \mathrm{kg}$, iv) was administered in $5 \mathrm{~min}$. O: main outcome: efficient rate S: Randomized controlled trial.

Condition being studied: There have been many randomized controlled trials to prove the clinical efficacy of Guanfu base $A$, but there is a lack of large-scale multicenter randomized controlled trials.

Information sources: Electronic databases such as PubMed, Embase web of science or Cochrane library, trial registers such as clinicaltrials.gov.

INPLASY registration number: This protocol was registered with the International Platform of Registered Systematic Review and Meta-Analysis Protocols (INPLASY) on 22 March 2021 and was last updated on 22 March 2021 (registration number INPLASY202130077).

\section{INTRODUCTION}

Review question / Objective: P: Patients with arrhythmia including patients with supraventricular or ventricular arrhythmia or patients with ventricular arrhythmia without severe structural heart disease or other diseases. I/C: Guanfu base $(4 \mathrm{mg} / \mathrm{kg}$, iv) A or propafenone (PRO, $1 \mathrm{mg} / \mathrm{kg}$, iv) was administered in $5 \mathrm{~min}$. $O$ : main outcome: efficient rate S: Randomized controlled trial.

Condition being studied: There have been many randomized controlled trials to prove the clinical efficacy of Guanfu base A, but 
there is a lack of large-scale multicenter randomized controlled trials.

\section{METHODS}

Participant or population: Patients suffered arrhythmia.

Intervention: Guan-fu base $A$ injection (4mg/kg).

Comparator: Propafenone (pro,1mg/kg).

Study designs to be included: There was no statistically significant difference in baseline between the test group and the control group. Randomized controlled trial. Have a clear outcome indicator. Have clear interventions.

Eligibility criteria: 1 . The subjects of the study were all arrhythmia patients. 2. Without heart structural diseases. 3 . The experimental group and the control group are clear. 4. Intervention measures are Guanfu A Hydrochloride Injection and Propafenone. 5. The type of this study is randomized controlled trial (RCT).

Information sources: Electronic databases such as PubMed, Embase web of science or Cochrane library, trial registers such as clinicaltrials.gov.

Main outcome(s): Efficient rate. Objective to observe Guan-fu base A effect of Propofol for cardioversion comparing with propafenone in $40 \mathrm{~min}$.

Quality assessment / Risk of bias analysis: This study followed the requirements of the international meta-analysis writing guidelines (the PRISMA statement for reporting systematic reviews and metaanalyses of studies that evaluate healthcare interventions: explanation and elaboration). Using the review manager 5.3 to evaluate literature quality.

Strategy of data synthesis: pooled effect, random effect, $R R$, OR.

Subgroup analysis: Perform subgroup analysis of documents with strong heterogeneity and find out the reasons for the existence of possible heterogeneity. Subgroup analysis for supraventricular tachycardia and ventricular tachycardia.

Sensitivity analysis: Draw a funnel chart and perform Begg and egger tests to determine whether the article is biased.

Country(ies) involved: China.

Keywords: Acehytisine Hydrochloride, Propafenone, Tachycardia, meta-analysis.

Contributions of each author:

Author 1 - Song Jinming.

Email: 928128995@qq.com

Author 2 - Tang Yao.

Email: tangyao1236789@163.com

Author 3 - Gao Chao.

Author 4 - Xu Zongpei. 